\title{
The Hospital Zambrano Hellion venous thromboembolism rapid response team (PREVENTION-team): Improving pulmonary embolism and deep venous thrombosis patient care
}

\section{Equipo de respuesta rápida para tromboembolismo venoso del Hospital Zambrano Hellion (PREVENTION-Team): Mejorando el manejo del tromboembolismo pulmonar y trombosis venosa profunda}

\author{
Raúl del Toro-Mijares ${ }^{1,2}$, Carlos Jerjes-Sánchez ${ }^{1,2,3 *}$, David Rodríguez ${ }^{2,3}$, Jathniel Panneflek ${ }^{3}$, \\ Mauricio Vázquez-Guajardo ${ }^{1,2}$, Mario A. Fabiani ${ }^{1,2}$, Juan Quintanilla ${ }^{1,2}$, Luis Manautou ${ }^{1,2}$, \\ Erasmo de la Peña-Almaguer ${ }^{1,2,3}$, Arturo Cadena ${ }^{1,2}$, Gabriela Cassagne ${ }^{2}$ y Guillermo Torre-Amione Cl,2,3,4 $^{1}$ \\ ${ }^{1}$ Escuela de Medicina y Ciencias de la Salud, Tecnológico de Monterrey, Monterrey; ${ }^{2}$ Instituto de Cardiología y Medicina Vascular; ${ }^{3}$ Centro de \\ Investigación Biomédica, Hospital Zambrano Hellion, TecSalud, San Pedro Garza García, Nuevo León, México; ${ }^{4}$ Methodist DeBakey Heart and \\ Vascular Center, The Methodist Hospital, Houston, Texas, USA
}

\begin{abstract}
Background: Fast-track worldwide reperfusion programs improve outcomes in ST-elevation myocardial infarction and stroke. Similar programs called Program Evaluation and Review Technique (PERT) focus on submassive and massive pulmonary embolism (PE) excluding deep venous thrombosis (DVT). Methods: PREVENTION-team (Hospital Zambrano Hellion Venous Thromboembolism [VTE] Rapid Response). Primary objective: Fast-track stratification, diagnostics, and treatment (60$90 \mathrm{~min}$ ) to improve proximal DVT and submassive and massive PE patients care. Secondary objectives: Increase diagnosis rate of low-risk PE and distal DVT; exploration of cause; long-term anticoagulation; identify high-risk profile for chronic complications; community-based support groups and patient education to extend the concept of the thrombosis-free hospital to thrombosis-free home. Structure and organization: The team includes cardiologists, vascular medicine, angiologist, echocardiographer, cardiovascular imaging, and interventional cardiologists. The team will be accessible $24 h$ a day, 7 days a week, 365 days a year, and base on previous national experience. The cardiology fellow on call will be responsible for activation and evaluation. We will design several tools to accelerate these processes. Risk stratification and therapeutic approach will be based on clinical presentation, echocardiogram, and biomarkers findings. According to PERT stratification based on resources and medical specialties, Hospital Zambrano Hellion has level 1 PERT. PREVENTION-team links physicians with different expertise, provide fast, efficient, and time-saving treatment, potentially saving lives and reducing bleeding and chronic complications in VTE patients. Finally, establishing a network in our hospital and health system to improve VTE patients care. To the best of our knowledge, this is the first rapid response team focused on VTE in Mexico.
\end{abstract}

Key words: Venous thromboembolism. Pulmonary embolism. Deep vein thrombosis. Rapid response teams. Program evaluation and review technique. Mexico.

Correspondencia:

${ }^{*}$ Carlos Jerjes Sánchez

E-mail: carlos.jerjes@udicem.org.mx

DOI: 10.24875/ACM.19000276
Disponible en internet: $30-01-2020$ Arch Cardiol Mex. 2020;90(1):28-38 www.archivoscardiologia.com 2604-7063/@ 2019 Instituto Nacional de Cardiología Ignacio Chávez. Publicado por Permanyer. Este es un artículo open access bajo la licencia CC BY-NC-ND (http://creativecommons.org/licenses/by-nc-nd/4.0/). 


\section{Resumen}

Antecedentes: Programas de reperfusión mejoraron la evolución en infarto con elevación del ST y accidente cerebrovascular embólico. Programas similares llamados PERT para TEP masiva o submasiva excluyen TVP. Métodos: Equipo PREVENTION (Hospital Zambrano Hellion Venous Thromboembolism Rapid Response). Objetivo primario: Estratificación, diagnóstico y tratamiento acelerado (60-90 minutos) para mejorar atención del TVP proximal y TEP masiva o submasiva. Objetivos secundarios: Incrementar diagnóstico de TEP de riesgo bajo y TVP distal; explorar causa; anticoagulación a largo plazo; perfil de riesgo alto para complicaciones crónicas; grupos de soporte en la comunidad y educación para pacientes, y extender el concepto de hospital libre de trombosis a hogar libre de trombosis. Estructura y organización: Incluye cardiólogos, medicina vascular, angiólogo, ecocardiografistas, imagen cardiovascular. Basado en experiencia nacional, el equipo estará accesible 24 horas del día, siete días de la semana, 365 días del año. El residente de cardiología realizará la activación y estratificación. Diseñamos herramientas para acelerar el proceso. La estratificación de riesgo y el abordaje terapéutico se basará en presentación clínica, hallazgos ecocardiograficos y biomarcadores. El Hospital Zambrano Hellion tiene nivel PERT 1 de acuerdo a la estratificación PERT basada en recursos y especialidades. Equipo-PREVENTION en TEV vincula médicos con diferentes capacidades, ofrece rápido y eficiente tratamiento para preservar vidas y reducir complicaciones hemorrágicas y crónicas. En nuestro hospital y sistema de salud establecer una sólida red de trabajo para mejorar la atención. Hasta nuestro conocimiento, en México este podría ser el primer equipo de respuesta rápida enfocado en TEV.

Palabras clave: Tromboembolismo venoso. Tromboembolia pulmonar. Trombosis venosa profunda. Equipos de respuesta rápida. PERT. México.

\section{Introduction}

Venous thromboembolism (VTE), including deep venous thrombosis (DVT) and pulmonary embolism (PE), is a worldwide disease characterized by cardiovascular mortality, impaired quality of life and significant longterm complications such as recurrence, a chronic thromboembolic pulmonary disease with or without pulmonary hypertension, and post-thrombotic syndrome (PTS) ${ }^{1}$. PE - the most severe consequence - is the third cause of cardiovascular mortality after myocardial infarction and stroke, the leading preventable cause of death in hospitalized patients, the main cause of pregnancy-related maternal death in developed countries, and the second cause of mortality in cancer patients ${ }^{1}$. Furthermore, VTE is the third most common complication in trauma patients, and PE is the third most common cause of death in patients who survive the first $24 \mathrm{~h}$ after injury ${ }^{2}$. PE survivors commonly have persistent right ventricle dysfunction, impaired functional status (NYHA Class II-IV), diminished exercise capacity (6-min walk test), and reduced quality of life in the follow-up ${ }^{3}$. In addition, 3.8\% are predicted to develop chronic thromboembolic pulmonary hypertension ${ }^{4}$.

On the other hand, up to $70 \%$ of patients with PE have DVT, and up to $32 \%$ of patients with DVT have asymptomatic $\mathrm{PE}^{5,6}$. Furthermore, PTS can be observed in up to $25-50 \%$ of DVT cases, of which, $5-10 \%$ later have severe limitations and poor quality of life. In addition, PTS exponentially increases health-care costs in the United States and Canada 7 . Despite this evidence, advanced therapies to reduce PTS incidence are not carried out expeditiously. Recently, Heart Teams are launched to improve the management of complex cardiovascular diseases ${ }^{8}$, including PE patients. In 2012, the Massachusetts General Hospital (MGH) created the first formal and successful multidisciplinary rapidresponse team, called program evaluation and review technique (PERT), to assess and provide clinical recommendations for patients with submassive and massive PE in real time ${ }^{9}$. Worldwide institutions reproduced similar concepts, mobilizing multidisciplinary teams that coordinate and provide optimal therapeutic options, which in turn improve patient care ${ }^{10}$. However, PERTs does not include DVT - the source of PE - despite the negative impact it has in terms of quality of life and public health $\operatorname{costs}^{11}$. We designed to improve the quality of care ${ }^{12}$ of the entire clinical spectrum of VTE, the first - to the best of our knowledge - rapid response team in Mexico, called Hospital Zambrano Hellion VTE Rapid Response Team (PREVENTION-team).

\section{Materials and methods}

\section{PREVENTION-team objectives}

Primary objective: to provide fast-track stratification and diagnostics (60-90 $\mathrm{min}$ ) after protocol activation to initiate anticoagulation alone promptly or anticoagulation plus advanced therapy (systemic or mechanical thrombolysis) in submassive, massive, and proximal DVT. The decision-making between anticoagulation alone or 


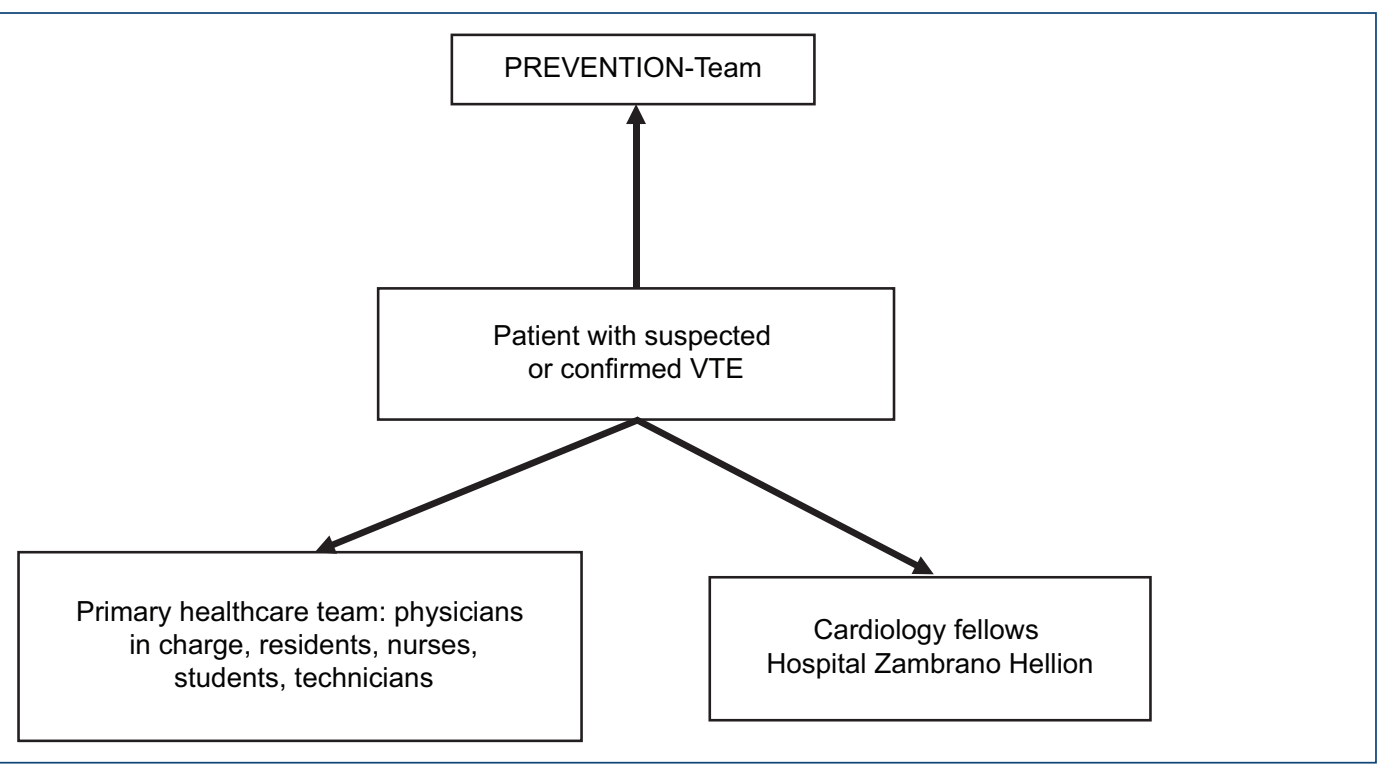

Figure 1. The PREVENTION-team is a patient-centered three-arm team where collaboration and effective communication is key for protocol success. VTE: venous thromboembolism.

advanced therapy will be by an experienced clinician and depends on the extension of the thrombus burden and right ventricular dysfunction severity. Secondary objectives: (1) in-hospital increased rate of low-risk PE and distal VTE patients; (2) exploration into the cause of PE as ensuring age-specific cancer-related screening, thrombophilia testing in patients $<40$ years with weak triggers, thrombus in unusual sites, or strong family history ${ }^{10}$; and (3) long-term anticoagulation management: election and length of anticoagulation, adherence, bleeding complications, and management. (4) To identify those with a high-risk profile for chronic thromboembolic disease, post-PE syndrome, chronic thromboembolic pulmonary hypertension, and PTS patients ${ }^{10}$. (5) To implement a prospective registry on Research Electronic Data Capture, an online platform high-quality surveys, and databases from Vanderbilt University supported by the National Institutes of Health (https://projectredcap. org/). (6) Organize community-based support groups, and patient education to improve adherence, to reduce recurrence, and bleeding complications, with de intention of extending the concept of the thrombosis-free hospital to thrombosis-free home.

\section{PREVENTION-team: structure and organization}

The multidisciplinary team includes physicians trained in cardiology, vascular medicine, angiology, echocardiography, cardiovascular imaging, and interventional cardiology. The team cornerstone will be the health-care team in charge (physician, nurses, residents, students, and technicians), cardiology fellows, and PREVENTION-team. Furthermore, effective coordination and communication will be mandatory for a successful program (Fig. 1). The team must be easily accessible and provide a consistent, rapid, and effective multidisciplinary response in the emergency room, intensive critical care unit, or in-hospital setting. The PREVENTION-team organization ensures a fast-track program to start specific treatment between 60 and 90 min after code activation, reproducing ST-elevation myocardial infarction, and ischemic stroke reperfusion programs.

\section{PREVENTION-team: activation and execution}

Table 1 shows the principal steps and the staff involved in the execution of the program. The first step of activation, which is based on clinical presentation (sudden dyspnea, near or syncope, chest pain such as angina, respiratory distress, and hypoxemia) suggests submassive or massive $\mathrm{PE}^{13}$ or proximal DVT (leg pain and swelling). Therefore, the hospital staff must know the VTE risk factors and how to identify high-clinically suspicious patients. Before the official launch, we will conduct educational programs, round table discussions, 
Table 1. Key steps, events, and personnel in the execution of the PREVENTION-team protocol

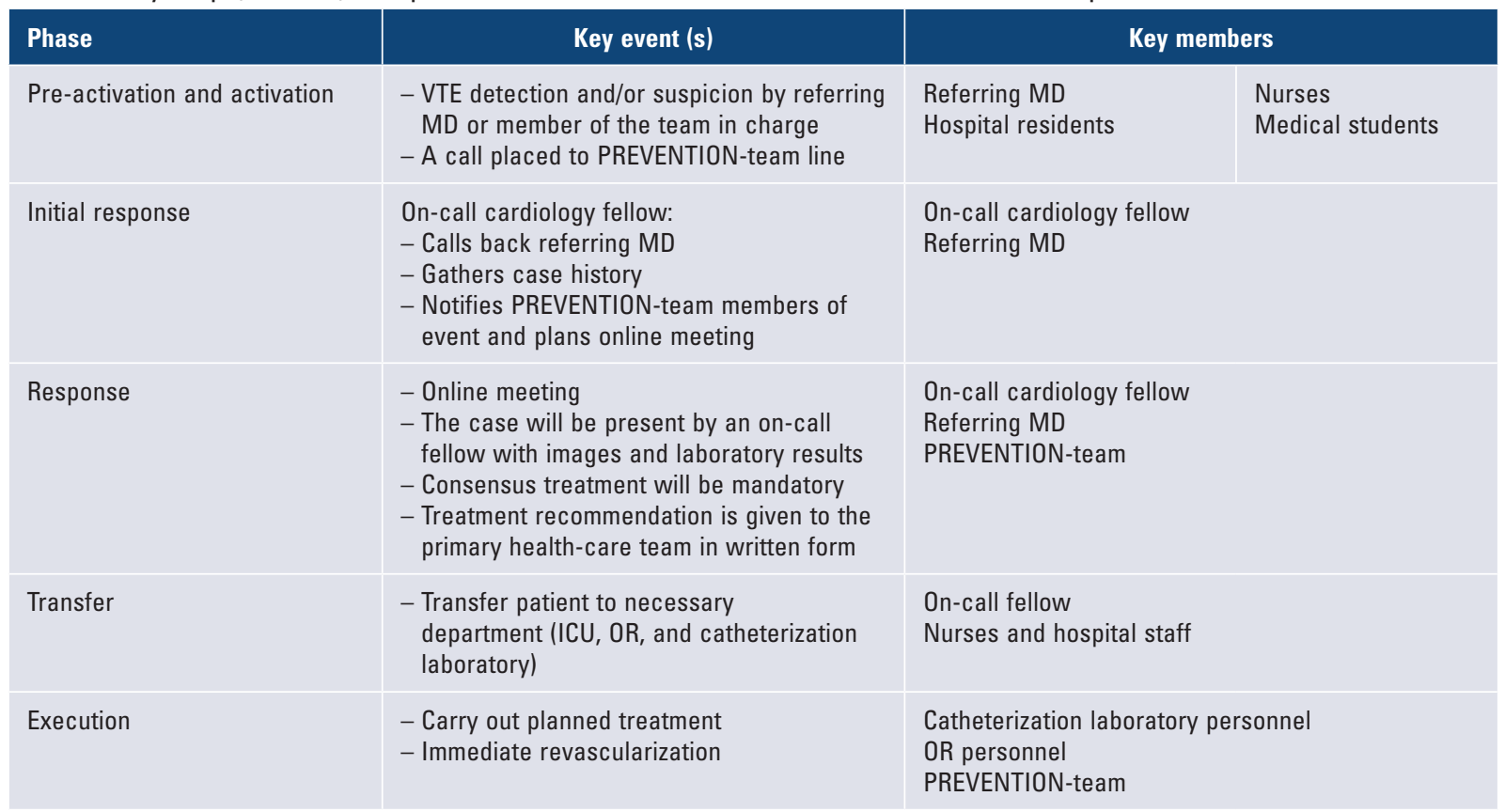

VTE: venous thromboembolism; ICU: intensive care unit; OR: operating room.

and case simulations geared toward hospital physicians, nurses, residents, students, and technicians. Furthermore, patient education will be mandatory to improve the outcome and reduce recurrence and bleeding complications in the follow-up.

An activation line will be available $24 \mathrm{~h}$ a day, 7 days a week, and 365 days a year. The cardiology fellow on call will be responsible for the protocol activation, immediate patient evaluation, and obtain imaging and laboratory studies to accelerate the diagnostic process and save time. This information will be present during an online meeting. The checklist called $\mathrm{S}_{2} \mathrm{HIELD}_{\mathrm{B}}$ (S signs and symptoms, $\mathbf{H}$ history, I image, $\mathbf{E}$ Electrocardiography, $\mathbf{L}$ laboratory, $\mathbf{D}$ demographics, and $\mathbf{B}$ bleeding risk) provides the team with the necessary information to establish a high-clinical suspicion, diagnosis, bleeding risk, and decision-making (Table 2). Risk stratification will be base on clinical presentation, echocardiogram, and biomarkers findings. Imaging techniques and or ultrasound will prove the final diagnosis.

After protocol activation, the on-call cardiology fellow will reach out to the PREVENTION-team through an electronic message. The team will be ready to hold an online conference as soon as possible (30 min), providing the on-call fellow enough time to assess the patient and obtain enough data to prove VTE accurately and $\mathrm{PE}$ diagnosis, quantify the venous thrombus burden and assess right ventricular dysfunction severity. Finally, within $60-90$ min of the initial call, a treatment recommendation will be issued to the physician in charge. The program will follow-up on the clinical condition, treatment response, and in-hospital complications to consistently improve patient care. All information, including clinical data, risk factors, clinical presentation, electrocardiogram (ECG), chest X-ray, biomarkers, diagnosis studies, as well as, therapeutic approach, will be captured in an electronic database. On discharge, patients will have a follow-up in the outpatient clinic if the healthcare team deems it necessary.

We considered 60-90 min as a window based on (1) our previous experience ${ }^{14-20}$, in which we perform stratification, diagnosis, and systemic thrombolysis in the first 90 min after PE patients arrive at the emergency room $^{15}$; (2) thrombus resistance ${ }^{21}$, right ventricular ischemia, and myocardial infarction ${ }^{17}$ are all time-dependent; and finally, (3) evidence from mechanical and pharmacological reperfusion in ST-elevation myocardial infarction and ischemic stroke programs ${ }^{11,22-24}$. Furthermore, we will activate the cardiac catheter lab and transesophageal echocardiography units in specific cases. According to PERT MGH hospital stratification based on resources and medical specialties ${ }^{9}$, Hospital Zambrano Hellion has Level 1 PERT; in other words, we have all the resources necessary to carry out a successful program. Recently, 
Table 2. $\mathrm{S}_{2} \mathrm{HIELD}_{\mathrm{B}}$ : Mandatory data to collected after PREVENTION activation

\begin{tabular}{|c|c|c|c|}
\hline \multirow{2}{*}{$\begin{array}{l}\text { Date: } \\
\text { Allergies: Yes/No }\end{array}$} & \multicolumn{2}{|c|}{ Time of activation/initial evaluation:/ } & Age and sex: \\
\hline & Days in hospital: & \multicolumn{2}{|c|}{ Days symptomatic in hospital/at home: } \\
\hline \multicolumn{2}{|l|}{ Signs and symptoms } & \multicolumn{2}{|l|}{ History } \\
\hline $\begin{array}{l}\text { Signs } \\
\text { Systolic blood pressure: } \\
\text { Heart rate: } \\
\mathrm{O}_{2} \text { saturation: } \\
\text { Symptoms } \\
\text { Assess DVT: } \\
\text { Lower limb pain: Yes/No } \\
\text { Swelling: Yes/No } \\
\text { Erythema: Yes/No } \\
\text { Homans sign: Yes/No } \\
\text { Ollow sign: Yes/No }\end{array}$ & $\begin{array}{l}\text { Assess PE: } \\
\text { Dyspnea: Yes/No } \\
\text { Ischemic like chest pain: Yes/No } \\
\text { Near or syncope: Yes/No } \\
\text { Cardiac arrest: Yes/No } \\
\text { Assess paradoxical embolism: } \\
\text { Headache: Yes/No } \\
\text { Back pain: Yes/No } \\
\text { Abdominal pain: Yes/No } \\
\text { Paresthesia: Yes/No }\end{array}$ & $\begin{array}{l}\text { VTE: Yes/No } \\
\text { Obesity: Yes/No } \\
\text { Recent infection: Yes/No } \\
\text { Puerperium recent: Yes/No } \\
\text { Pregnancy: Yes/No } \\
\text { Major surgery recent: Yes/No } \\
\text { Minor surgery recent: Yes/No } \\
\text { Prolonged bed rest/trip: Yes/No } \\
\text { Estrogen/OCP use: Yes/No } \\
\text { Known active cancer: Yes/No }\end{array}$ & \\
\hline \multicolumn{4}{|c|}{ Imaging } \\
\hline $\begin{array}{l}\text { Chest X-ray } \\
\text { Westermark sign: Yes/No } \\
\text { PA amputation: Yes/No }\end{array}$ & $\begin{array}{l}\text { Echocardiogram } \\
\text { RV dilation: Yes/No } \\
\text { McConnell sign: Yes/No } \\
\text { In-transit thrombus:Yes/No }\end{array}$ & $\begin{array}{l}\text { CT angiogram } \\
\text { Size: } \\
\text { Location: } \\
\text { Burden thrombus: }\end{array}$ & $\begin{array}{l}\text { Lower limb } \\
\text { Doppler US } \\
\text { Thrombus: Yes/No } \\
\text { Location: distal/ } \\
\text { Proximal } \\
\text { Burden Thrombus: } \\
\text { Floating thrombus: } \\
\text { Yes/No }\end{array}$ \\
\hline \multicolumn{2}{|r|}{ ECG } & \multicolumn{2}{|l|}{ Laboratory } \\
\hline $\begin{array}{l}\text { Tachycardia: Yes/No } \\
\text { Atrial fibrillation or flutter: } \\
\text { Yes/No } \\
\text { RBBB: Yes/No } \\
\text { S103T3: Yes/No }\end{array}$ & $\begin{array}{l}\text { ST dynamic changes: Yes/No } \\
\text { aVR ST elevation: Yes/No } \\
\text { V1 qR and ST elevation: Yes/No } \\
\text { RV strain overload: Yes/No }\end{array}$ & $\begin{array}{l}\text { Hemoglobin: } \_ \text {Platelets: } \\
\text { BNP: } \_ \text {D-dimer: } \\
\text { High-sensitivity troponin I: } \\
\text { eGFR: }\end{array}$ & \\
\hline \multicolumn{4}{|c|}{ Bleeding risk } \\
\hline $\begin{array}{l}>65-75 \text { years: Yes/No } \\
\text { Female: Yes/No } \\
\text { BMI < } 24 \text { kg/m²: Yes/No } \\
\text { Weight < 50-60 kgs: Yes/No } \\
\text { Cancer: Yes/No } \\
\text { INR > 2.5: Yes/No }\end{array}$ & $\begin{array}{l}\text { Oral anticoagulation: Yes/No } \\
\text { Recent major surgery: Yes/No } \\
\text { Uncontrolled hypertension: Yes/No } \\
\text { eGFR < } 30 \mathrm{ml} \\
\text { Liver/kidney disease: Yes/No }\end{array}$ & $\begin{array}{l}\text { Bleeding predisposition: Yes/No } \\
\text { Alcohol abuse: Yes/No } \\
\text { Thrombocytopenia: Yes/No } \\
\text { History of stroke: Yes/No }\end{array}$ & \\
\hline
\end{tabular}

DVT: deep venous thrombosis; PE: pulmonary embolism; VTE: venous thromboembolism; OCP: oral contraceptive pills; PA: pulmonary artery; RV: right ventricle; RBBB: right bundle branch block; BNP: B-type natriuretic peptide; eGFR: estimated glomerular filtration rate; INR: international normalized ratio.

the impact of PERT MGH was demonstrated by a significant mortality reduction (25\%) in massive PE compared with the previous registries ${ }^{25}$. This evidence suggests that a rapid response team can modify in-hospital outcomes in a group of patients with high mortality risk.

\section{PREVENTION-team: therapeutic approach}

\section{Anticoagulation}

The foundation of VTE treatment is anticoagulation, and advanced therapy is the option in impending or clinically unstable patients. Table $3^{11,26,27}$ shows anticoagulation options in the acute phase, long-term, and extended phase. Unprovoked VTE, recurrence, active cancer, proved or strong suspicion of thrombophilia and a persistently abnormal D-dimer required long-term anticoagulation. In patients with DVT with or without $\mathrm{PE}$, we suggest low-molecular-weight heparin, enoxaparin instead of unfractionated heparin. Furthermore, non-Vitamin $\mathrm{K}$ antagonist oral anticoagulants are effective and possess a safer profile compared to Vitamin $\mathrm{K}$ antagonists (Table 3). Anticoagulation alone is recommended in low-risk PE patients (clinical stability, no biomarkers expression, without severe right ventricular dysfunction, and moderate thrombus burden); the route 
Table 3. Parenteral and oral anticoagulants ${ }^{11,26,36}$

\begin{tabular}{|l|l}
\hline Acute phase & Weight-adjusted unfractionated heparin \\
1. Unfractionated heparin $60 \mathrm{U} / \mathrm{kg}$ bolus (maximum $4000 \mathrm{U}$ ) followed by $12 \mathrm{U} / \mathrm{kg}$ \\
infusion (maximum $1000 \mathrm{U})$ \\
Standard unfractionated heparin regimen \\
2. Unfractionated heparin $80 \mathrm{U} / \mathrm{kg}$ bolus followed by $18 \mathrm{U} / \mathrm{kg} / \mathrm{h}$ infusion \\
Low-molecular-weight heparin \\
3. Enoxaparin intravenous bolus $30 \mathrm{mg}$ followed by subcutaneous injection $(1 \mathrm{mg} / \mathrm{kg}$ \\
BID or $1.5 \mathrm{mg} / \mathrm{kg}$ ONCE); in patients $>75$ years no bolus and $0.75 \mathrm{mg} / \mathrm{kg}$ BID \\
Non-vitamin K antagonist oral anticoagulants (NOACs) \\
4. Apixaban: $10 \mathrm{mg}$ twice daily for 7 days, followed by $5 \mathrm{mg}$ twice daily \\
5. Rivaroxaban: $15 \mathrm{mg}$ twice daily for 3 weeks, followed by $20 \mathrm{mg}$ daily \\
\hline Vitamin K antagonists \\
\hline Warfarin $5 \mathrm{mg}$ daily, overlapped with heparin for first 5 days until two consecutive \\
INR in therapeutic ranges (2-3), and then dose-adjusted to maintain INR $2-3$ \\
Low-molecular-weight heparin \\
In patients with active cancer: subcutaneous injection $40 \mathrm{mg}$ 0NCE \\
NOACs \\
Dabigatran: $150 \mathrm{mg}$ BID \\
Apixaban: $5 \mathrm{mg}$ or $2.5 \mathrm{mg} \mathrm{BID}$ \\
Rivaroxaban: $20 \mathrm{mg}$ or $15 \mathrm{mg}$ ONCE
\end{tabular}

NOACs: non-Vitamin K antagonist oral anticoagulants; INR: international normalized ratio.

of administration regimen and type will be up to the preference of the physicians in charge. In the extended phase, the low-molecular-weight heparin, enoxaparin, is indicated in active cancer patients. Unfractionated heparin is an option in severe kidney diseases, highrisk bleeding, $>75$ years, hypotension, impending clinical instability patients, and as adjunctive treatment ${ }^{11}$. We recommend enoxaparin in low-risk PE patients starting with an intravenous bolus, except in elderly patients in whom a dose reduction is mandatory $(\text { Table } 3)^{11}$. Loading apixaban or rivaroxaban doses are an effective and safe option in low-risk PE patients. In intermediate-risk, also called submassive $\mathrm{PE}$, we recommend weight-adjusted unfractionated heparin for the first 24-48 h, over enoxaparin to avoid heparin crossover if clinical status worsens. The use of unfractionated heparin as adjunctive treatment with a posterior switch to enoxaparin is a worldwide recommendation. This regimen was effective and safe, without intracranial hemorrhage in Mexican PE patients submitted to systemic thrombolysis ${ }^{15}$.

\section{Advanced therapy}

\section{DVt THROMBOLYSIS AND PERCUTANEOUS THROMBECTOMY}

Although there are not recommendations to systemic thrombolysis in iliofemoral DVT patients ${ }^{26}$, we recommend catheter-directed thrombolysis with alteplase at a dose of $0.01 \mathrm{mg} / \mathrm{kg} / \mathrm{h}$ (maximum $1 \mathrm{mg} / \mathrm{h}$ ) for iliofemoral DVT (Table 4) ${ }^{11}$. This therapeutic approach could reduce thrombus burden and venous hypertension, restore venous permeability, rescue limb in case of ischemia, and decrease PE risk. We also recommend percutaneous mechanical or pharmacomechanical thrombolysis. Various percutaneous devices are available with different mechanical principles for the removal of clot or thrombolysis: suction, rotation, rheolytic thrombectomy, and ultrasound ${ }^{28-31}$. The pharmacoinvasive approach combines the mechanical method and pharmacologic therapy to achieve thrombolysis ${ }^{32}$. This approach has shown to be effective with a lower dose of the thrombolytic drug and shorter procedural time with no difference in major bleeding or recurrence $^{33}$. As part of the thrombectomy procedure, we recommend the use of a prophylactic vena cava filter, as $17 \%$ of patients treated suffered asymptomatic PE demonstrated on computed tomography scans ${ }^{34}$. These filters should be removed as soon as possible ${ }^{35}$. When DVT occurs in the left iliac vein, we encourage the use of intravascular ultrasound to diagnose iliac compression (May-Thurner syndrome) ${ }^{36}$. If an iliac obstruction, residual thrombus or iliac stenosis is observed, angioplasty and dedicated vein stents use must be considered to improve patency ${ }^{28}$.

\section{Pe thrombolysis}

International and national guidelines ${ }^{26,27-38}$ recommend unfractionated heparin as adjunctive treatment and systemic thrombolysis in a well-selected (Table 5) ${ }^{11}$ 
Table 4. Anticoagulation and advanced therapy in venous thromboembolism patients $\mathbf{s}^{11,26,36,37,38}$

\begin{tabular}{|c|c|}
\hline $\begin{array}{l}\text { Distal deep venous } \\
\text { thrombosis }\end{array}$ & $\begin{array}{l}\text { Anticoagulation } \\
\text { Weight-adjusted unfractionated heparin } \\
\text { Unfractionated heparin } 60 \mathrm{U} / \mathrm{kg} \text { bolus (maximum } 4000 \mathrm{U} \text { ) followed by } 12 \mathrm{U} / \mathrm{kg} \text { infusion } \\
\text { (maximum } 1000 \mathrm{U} \text { ) } \\
\text { Standard unfractionated heparin regimen } \\
\text { Unfractionated heparin } 80 \mathrm{U} / \mathrm{kg} \text { bolus followed by } 18 \mathrm{U} / \mathrm{kg} / \mathrm{h} \text { infusion } \\
\text { Low-molecular-weight heparin } \\
\text { Enoxaparin intravenous bolus } 30 \mathrm{mg} \text { followed by subcutaneous injection }(1 \mathrm{mg} / \mathrm{kg} \mathrm{BID} \mathrm{or} 1.5 \mathrm{mg} / \mathrm{kg} \\
\text { ONCE); in patients > } 75 \text { years no bolus and } 0.75 \mathrm{mg} / \mathrm{kg} \mathrm{BID} \\
\text { Non-Vitamin K antagonist oral anticoagulants (NOACs) } \\
\text { Apixaban: } 10 \mathrm{mg} \text { twice daily for } 7 \text { days, followed by } 5 \mathrm{mg} \text { twice daily } \\
\text { Rivaroxaban: } 15 \mathrm{mg} \text { twice daily for } 3 \text { weeks, followed by } 20 \mathrm{mg} \text { daily }\end{array}$ \\
\hline $\begin{array}{l}\text { Proximal deep venous } \\
\text { thrombosis }\end{array}$ & $\begin{array}{l}\text { Adjunctive treatment } \\
\text { Weight-adjusted unfractionated heparin } \\
\text { Unfractionated heparin } 60 \mathrm{U} / \mathrm{kg} \text { bolus (maximum } 4000 \mathrm{U} \text { ) followed by } 12 \mathrm{U} / \mathrm{kg} \text { infusion (maximum } 1000 \mathrm{U} \text { ) } \\
\text { Standard unfractionated heparin regimen } \\
\text { Unfractionated heparin } 80 \mathrm{U} / \mathrm{kg} \text { bolus followed by } 18 \mathrm{U} / \mathrm{kg} / \mathrm{h} \text { infusion } \\
\text { Catheter-directed thrombolysis } \\
\text { Alteplase, } 0.01 \mathrm{mg} / \mathrm{kg} / \mathrm{h} \text { (maximum } 1 \mathrm{mg} / \mathrm{h} \text { ) } \\
\text { Ultrasound-facilitated catheter-directed thrombolysis (USCDT) }\end{array}$ \\
\hline Low-risk PE & $\begin{array}{l}\text { Anticoagulation } \\
\text { Weight-adjusted unfractionated heparin } \\
60 \mathrm{U} / \mathrm{kg} \text { bolus (maximum } 4000 \mathrm{U} \text { ) followed by } 12 \mathrm{U} / \mathrm{kg} \text { infusion (maximum } 1000 \mathrm{U} \text { ) } \\
\text { Low-molecular-weight heparin } \\
\text { Enoxaparin intravenous bolus } 30 \mathrm{mg} \text { followed by subcutaneous injection (1 mg/kg BID or } 1.5 \mathrm{mg} / \mathrm{kg} \\
\text { ONCE); in patients > } 75 \text { years no bolus and } 0.75 \mathrm{mg} / \mathrm{kg} \text { BID } \\
\text { Non-Vitamin K antagonist oral anticoagulants (NOACs) } \\
\text { Apixaban: } 10 \mathrm{mg} \text { twice daily for } 7 \text { days, followed by } 5 \mathrm{mg} \text { twice daily } \\
\text { Rivaroxaban: } 15 \mathrm{mg} \text { twice daily for } 3 \text { weeks, followed by } 20 \mathrm{mg} \text { daily }\end{array}$ \\
\hline $\begin{array}{l}\text { Intermediate risk/ } \\
\text { submassive PE with or } \\
\text { without impending clinical } \\
\text { instability }\end{array}$ & $\begin{array}{l}\text { Weight-adjusted unfractionated heparin } \\
60 \mathrm{U} / \mathrm{kg} \text { bolus (maximum } 4000 \mathrm{U} \text { ) followed by } 12 \mathrm{U} / \mathrm{kg} \text { infusion (maximum } 1000 \mathrm{U} \text { ) and close monitoring } \\
\text { of blood pressure, oxygen saturation, heart and respiratory rate (consider thrombolysis in case of } \\
\text { impending or hypotension or clinical instability) } \\
\text { Ultrasound-facilitated catheter-directed thrombolysis (USCDT) } \\
\text { USCDT } \times 2 \mathrm{~h} \text { with alteplase infusion at } 2 \mathrm{mg} / \mathrm{h} / \text { catheter (range } 4-8 \mathrm{mg} ; 1 \mathrm{vs.} 2 \text { lungs) }\end{array}$ \\
\hline $\begin{array}{l}\text { A high-risk or massive PE } \\
\text { or submassive PE with } \\
\text { impending clinical instability }\end{array}$ & $\begin{array}{l}\text { Adjunctive treatment } \\
\text { Weight-adjusted unfractionated heparin } \\
60 \mathrm{U} / \mathrm{kg} \text { bolus (maximum } 4000 \mathrm{U} \text { ) followed by } 12 \mathrm{U} / \mathrm{kg} \text { infusion (maximum } 1000 \mathrm{U} \text { )/24 h or } 48 \mathrm{~h} \text { followed } \\
\text { by enoxaparin } 1 \mathrm{mg} / \mathrm{kg} \text { BID or } 1.5 \mathrm{mg} / \mathrm{kg} \text { ONCE/5 days or apixaban or rivaroxaban } \\
\text { Systemic thrombolysis } \\
50 \mathrm{mg} \text { of alteplase in } 1-2 \mathrm{~h} \text { in }>60 \text { years } \\
100 \mathrm{mg} \text { of alteplase in } 1-2 \mathrm{~h} \text { in }<60 \text { years } \\
\text { Weight-adjusted tenecteplase bolus in }<60 \text { years: } 30 \mathrm{mg}<60 \mathrm{~kg}, 35 \mathrm{mg} 60-70 \mathrm{~kg}, 40 \mathrm{mg} 70-80 \mathrm{~kg}, 45 \mathrm{mg} \\
80-90 \mathrm{~kg}, 50 \mathrm{mg}>90 \mathrm{~kg} \\
\text { Catheter-directed thrombolysis } \\
30 \pm 10 \mathrm{mg} \text { of alteplase } \\
\text { Pharmacoinvasive approach } \\
\text { Thrombus fragmentation with pigtail catheter, } 20 \mathrm{mg} \text { alteplase infusion in the pulmonary artery and } \\
\text { manual or percutaneous aspiration with aspiration device (Aspirex or Pronto) } \\
\text { Ultrasound-facilitated catheter-directed thrombolysis (USCDT) } \\
\text { USCDT } \times 2 \mathrm{~h} \text { with alteplase infusion at } 2 \mathrm{mg} / \mathrm{h} / \mathrm{catheter} \text { (range } 4-8 \mathrm{mg} ; 1 \mathrm{vs.} 2 \text { lungs) }\end{array}$ \\
\hline $\begin{array}{l}\text { Absolute contraindicati } \\
\text { for anticoagulation or } \\
\text { thrombolysis }\end{array}$ & $\begin{array}{l}\text { Venous cava filter } \\
\text { Removed temporary filters between day } 24 \text { and } 54 \text { after placement. }\end{array}$ \\
\hline
\end{tabular}

high-risk or massive PE patient (IIb). European and American College of Chest Physicians ${ }^{27,37}$ recommendations are against thrombolysis in intermediate highrisk or submassive $P E$ patients because of the increased rate of intracranial hemorrhage ${ }^{39}$. The PEITHO study $^{40}$ and additional previous evidence have shown in-hospital improvement outcome, with systemic thrombolysis $^{14,16,17,19}$ in this group. Considering current and previous evidence, we recommended weight-adjusted unfractionated heparin as adjunctive treatment and systemic thrombolysis (IIB) in a well-selected high-risk or massive PE patient. We recommend half dose 
Table 5. Absolute contraindications for thrombolysis ${ }^{11}$

\begin{tabular}{|c|c|}
\hline $\begin{array}{l}\text { Previous intracranial } \\
\text { hemorrhage } \\
\text { Structural cerebrovascular } \\
\text { disease } \\
\text { Intracranial malignant neoplasm } \\
\text { Active bleeding } \\
\text { lespecially gastrointestinal in } \\
\text { last } 30 \text { day }\end{array}$ & $\begin{array}{l}\text { Aortic dissection or } \\
\text { suspicion of } \\
\text { Recent cranial surgery or } \\
\text { facial trauma with evidence } \\
\text { of fracture or cerebral } \\
\text { lesion } \\
\text { INR }>2.5 \\
\text { Stroke in past } 3 \text { months }\end{array}$ \\
\hline
\end{tabular}

INR: international normalized ratio.

Table 6. Therapeutic alternatives in high-risk bleeding patients ${ }^{11,42}$

\begin{tabular}{l|l}
$\begin{array}{l}\text { Low-dose catheter-directed } \\
\text { thrombolysis (alteplase } 20-40 \mathrm{mg} \text { ) }\end{array}$ & $\begin{array}{l}\text { Invasive pharmacological } \\
\text { treatment with thrombi } \\
\text { fragmentation and } \\
\text { aspiration }\end{array}$ \\
$\begin{array}{l}\text { OPTALYSE study: treatment arm } \\
1 \text { (alteplase } 2 \mathrm{mg} / \text { lung/2 } \mathrm{h} \text { ) and } \\
\text { treatment arm } 2 \text { (alteplase } 2 \mathrm{mg} /\end{array}$ & $\begin{array}{l}\text { Surgical embolectomy } \\
\text { lung/4 } \mathrm{h} \text { ) }\end{array}$
\end{tabular}

short-term alteplase infusion (Table 4), instead tenecteplase in patients over 60 years considering the high incidence of intracranial hemorrhages, especially in female patients. At present, to the best of our knowledge, half dose short-term alteplase infusion has no evidence of intracranial hemorrhage in the elderly population ${ }^{40}$. Furthermore, we recommend 1 or $2 \mathrm{~h} 100 \mathrm{mg}$ alteplase infusion or tenecteplase in a bolus in patients $<60$ years. Avoid unnecessary venous or arterial punctures to reduce major or minor bleeding complications. Systemic thrombolysis would be an important therapeutic option in intermediate-high-risk or submassive PE with impending clinical instability ${ }^{11}$ defined with at least one: oxygen desaturation $<90 \%$, respiratory distress, blood pressure in lower limits, advanced degree right branch block, severe global right ventricular hypokinesis, tricuspid annular plane systolic excursion $<13 \mathrm{~mm}$, high measurements of cardiac I troponin high-sensitivity, and B-type natriuretic peptide.

We recommend pharmacoinvasive therapy in patients with intermediate- or high-risk bleeding complications since this therapeutic approach showed efficacy and safety in the Mexican population ${ }^{41}$. Recently, the OPTALYSE trial ${ }^{42}$ significantly reduced alteplase dose and procedure time compared with previous ultrasound-facilitated catheter-directed thrombolysis studies ${ }^{43,44}$ (Table 6). The OPTALYSE approach improves the inadmissible long-term infusions $(\sim 12 \mathrm{~h})$ in cardiogenic shock or low cardiac output syndrome patients through a lowdose ultrasound-facilitated catheter-directed thrombolysis. Although alteplase $2 \mathrm{mg}$ in 2-h short infusion had no major bleeding complications in a broad clinical PE spectrum, including submassive PE patients, we will recommend $4 \mathrm{mg}$ to obtain a better reperfusion ${ }^{42}$ (Table 4). Finally, we recommend temporary inferior vena cava filters in patients with absolute contraindications for anticoagulation and thrombolysis in probed proximal DVT with or without in-transit thrombus patients ${ }^{11}$ (Table 4).

Patent foramen oval (PFO) and clinical or subclinical paradoxical cerebral or systemic emboli are frequent and an underestimated complication in submassive and massive PE patients ${ }^{45}$. Transesophageal echocardiogram identifies a high incidence of PFO (56\%) and cerebral magnetic resonance a high incidence $(17 \%)$ of subclinical ischemic stroke-related with a large shunt in submassive PE patients. Hemorrhagic transformation of subclinical ischemic stroke ${ }^{45}$ could explain unexpected intracranial hemorrhages after anticoagulation alone or advanced therapy in PE patients. Transthoracic echocardiogram with peripheral intravenous agitated saline bubbles to screen for PFO is mandatory ${ }^{45}$. PREVENTION-team should look for symptoms or signs suggesting central or systemic embolism in the clinical evaluation of high-clinical suspicion PE patients (Table 2).

\section{Research and educational activities}

Members of the core team will be the steering committee for all PREVENTION-team activities. Secondary objectives include leading research protocols, creation of support groups, and expand our network. We will set into an online database that will store all information regarding demographics, clinical presentation, therapeutic and diagnosis approaches, as well as the overall outcome for further research and analysis. Expansion of the network will allow us to implement our system in another clinical setting, identify possible loopholes not evident at our hospital, and further improve awareness of VTE. The creation of support groups creates a feeling of identification among patients, improving their well-being and health care. The PREVENTION-team will hold monthly meetings to review protocol activations, assess the response of the team, and troubleshoot and address any system issues. Educational activities, such as clinical case presentations and discussions, teaching sessions, and case simulations, will be important to maintain program quality. 
Although the main target of the program is an optimal fast-track treatment in VTE patients, primary or secondary prevention to reduce incidence or recurrence will be mandatory. We have had in-hospital strategies (thrombosis-free hospital), such as thrombosis risk stratification and pharmacologic and no-pharmacologic primary prevention to reduce VTE events for many years; however, these kinds of strategies lack at home. We identified that over $70 \%$ of PE patients come from a hospital outside. Thus, we propose patient and family education to stimulate early VTE recognition, identify trigger factors, and implement secondary no-pharmacologic prevention to extend the concept of a thrombosis-free hospital to a new concept: a thrombosis-free home.

\section{Discussion}

VTE is a major health problem, annually affecting 108 people per $100,000^{46}$ and in the United States 300,000 $600,000^{47}$. The chronic complications of VTE increase mortality, decrease functional class and quality of life, and increase health-care costs. Patients with PTS increase the cost $(2-10 \text { billion dollars annually })^{47}$ in the United States (7000) and Canada (4527) compared with DVT patients ${ }^{7}$. PE is the cause of preventable in-hospital and home mortality through pharmacologic or non-pharmacologic primary or secondary prevention. Considering the link between DVT and $\mathrm{PE}^{5,6}$, its high recurrence ${ }^{48}$, and the high health systems costs ${ }^{7}$, DVT should not be underestimated ${ }^{48}$. In the PREVENTION-team, we include the broad clinical spectrum of VTE to perform a fast-track risk stratification, multimodal diagnosis, and treatment to improve PE and DVT patient care. Another important objective will also be to increase the detection of in-hospital and in the emergency room of low-risk PE patients, whose impact and prevalence are not well defined.

At present, national and international guidelines lack the strong class of recommendation and level of evidence (IA) and do not yet consider new therapeutic approaches ${ }^{11,26,27}$. Furthermore, there is an underuse of systemic thrombolysis even in high-risk PE due to a fear of bleeding complications, and we do not have any evidence or recommendations in octogenarian and nonagenarian patients. Although systemic thrombolysis is a dark zone in intermediate high-risk or submassive PE patients, those with impending clinical instability should be eligible. In addition, treatment decision-making more often is based on personal medical experience ${ }^{9}$ instead of consensus discussion among experts. We hope that PREVENTION-team unifies risk stratification, diagnosis, and therapeutic approach through a coordinated action among health system staff to improve the quality of VTE patient care in our hospital.

\section{The first "Mexican PERT"}

In 1993, we performed the first successful systemic thrombolysis in a massive PE patient in Mexico ${ }^{17}$, following the lesson from the first national program on systemic fibrinolysis in ST-elevation myocardial infarction in the emergency room at Cardiology Hospital of the National Medical Center, IMSS. Shortly after that, we launched a successful open-label, randomized control trial proving that short-term streptokinase infusion by peripheral vein compared with unfractionated heparin reduces mortality in cardiogenic shock and massive PE patients ${ }^{19}$. The emergency, nuclear medicine, and echocardiography teams were activated quickly and efficiently, and systemic thrombolysis was delivered in the first 90 min after patient arrival at the emergency room department. The next challenge was to reproduce this approach $24 \mathrm{~h}$ a day, 365 days a year. All emergency physicians received training in echocardiography to improve patient care, and in the case of $V / Q$ lung scan unavailability, in high clinical suspicion patients with clinical, ECG, and echocardiographic findings of severe pulmonary hypertension and right ventricular dysfunction with impending clinical instability or hypotension, an experienced physician administered thrombolysis by peripheral vein. Following this strategy, we perform successful systemic thrombolysis in $11 \mathrm{PE}$ patients ${ }^{16}$. European Cardiology Society guidelines provide for this approach IC evidence level ${ }^{27}$.

In another hand, the process was very slow and complicated in submassive PE patients. In this group, although echocardiogram aided in the evaluation of the right ventricular function, the support of the departments of echocardiography and nuclear medicine had delays of up to $24 \mathrm{~h}^{16}$. Hence, we launched a working group, including physicians of the emergency room department and heads of echocardiography and nuclear medicine departments. After several meetings, we were able to agree in a significant reduction $(90 \mathrm{~min})$ of the time needed to perform the studies. Eight years later, in 2009, we perform our second $24 \mathrm{~h}$ a day, 365 days a year fast-track program for thrombolysis in submassive, impending clinical instability, and massive PE patients ${ }^{15}$. In this period, we perform under high clinical suspicion, $E C G$, and echocardiography findings successful thrombolysis in $8 \mathrm{PE}$ patients. The time to perform risk stratification, diagnosis, and systemic thrombolysis treatment were around $60 \mathrm{~min}$ in submassive and massive $\mathrm{PE}^{15}$. 


\section{The need to improve PE patient care}

Nineteen years after our fast-track programs for thrombolysis in massive $\mathrm{e}^{19}$ and submassive ${ }^{16} \mathrm{PE}$ patients, the first formal PERT ${ }^{9}$ emerged as a priority need to deliver rapid assessment and treatment of patients whose clinical condition is deteriorating but are not yet in shock or cardiac arrest ${ }^{49}$. The PERT consortium was then officially inaugurated in 2015, to create a sense of community where the different PERT programs across the world could share their experiences and work together and improve patient care. The design of our program is based on the already existing protocols and in our 24 years of experience ${ }^{14-20}$. We add some characteristics obtained from our experience with the entire clinical spectrum of VTE in mind what increases the quality of the program. With the implementation of the PREVENTION-team program, we look to provide fast, efficient, and time-saving treatment, potentially preserving lives and reducing bleeding and chronic complications in VTE patients. Furthermore, we will try to increase detection of low-risk PE. The success of the program will be the rapid and efficient communication among paramedic staff, technicians, residents, fellows, medical students, and physicians. Furthermore, the proper layout, functioning, and usage of the resources will be determinant in protocol success.

Finally, the activation of the PREVENTION-team program will pave the way for research and educational activities, including grand rounds, innovation projects, teaching sessions, and case discussion. Furthermore, another objective is to inspire consciousness among the hospital community, creating a learning environment and experience for everyone, and increasing the quality program. At present, although we have strategies including risk questionnaires at admission, compression stockings, or primary pharmacologic prevention to prevent in-hospital VTE, preventive strategies are lacking at home for patients with the same VTE high-risk. Finally, considering these observations, it is mandatory to extend the concept of the thrombosis-free hospital to the thrombosis-free home.

VTE is a very common cardiovascular disease with high morbidity and mortality, increased health-care costs, and complex treatment. Current VTE treatment is not standardized and depends on individual decisions of several medical specialties related to patient care (pulmonologists, cardiologists, internal medicine, surgeons, etc.). In addition, to the best of our knowledge, there are no available fast-track advanced programs in Mexico. Furthermore, international and national guidelines for PE management ${ }^{11,26,27}$ do not include a specific door-to needle timeframe to initiate advanced therapy, even though right ventricular ischemia and thrombus resistance are time dependent. Therefore, it is imperative to improve health care through an expert-conformed multidisciplinary team to fast-track stratification and optimal treatment to improve the outcome and long-term complications of VTE patients. PREVENTION-team links a group of physicians with different areas of expertise, guaranteeing collective, and synchronized medical care. We hope to create a strong network and inspire physicians, fellows, residents, nurses, medical students and staff of our hospital and health system not only to be aware of the problem but also enrich themselves with the necessary tools to diagnose and offer the best treatment possible.

\section{Conflicts of interest}

None.

\section{Funding}

No funding.

\section{Ethical disclosures}

Protection of human and animal subjects. The authors declare that no experiments were performed on humans or animals for this study.

Confidentiality of data. The authors declare that they have followed the protocols of their work center on the publication of patient data.

Right to privacy and informed consent. The authors declare that no patient data appear in this article.

\section{References}

1. Vazquez-Garza E, Jerjes-Sanchez C, Navarrete A, Joya-Harrison J, Rodriguez D. Venous thromboembolism: thrombosis, inflammation, and immunothrombosis for clinicians. J Thromb Thrombolysis. 2017; 44:377-85.

2. Miano TA, Cuker A, Christie JD, Martin N, Smith B, Makley AT, et al. Comparative effectiveness of enoxaparin vs dalteparin for thromboprophylaxis after traumatic injury. Chest. 2018;153:133-42.

3. Sista AK, Miller LE, Kahn SR, Kline JA. Persistent right ventricular dysfunction, functional capacity limitation, exercise intolerance, and quality of life impairment following pulmonary embolism: systematic review with meta-analysis. Vasc Med. 2017;22:37-43.

4. McNeil K, Dunning J. Chronic thromboembolic pulmonary hypertension (CTEPH). Heart. 2007;93:1152-8.

5. Kearon C. Natural history of venous thromboembolism. Circulation. 2003;107:122-30.

6. Dalen JE. Pulmonary embolism: what have we learned since Virchow? Natural history, pathophysiology, and diagnosis. Chest. 2002;122:1440-56.

7. Kahn SR, Comerota AJ, Cushman M, Evans NS, Ginsberg JS, Goldenberg NA, et al. The postthrombotic syndrome: evidence-based prevention, diagnosis, and treatment strategies: a scientific statement from the American heart association. Circulation. 2014;130:1636-61. 
8. Zern EK, Young MN, Rosenfield K, Kabrhel C. A pulmonary embolism response team: initial experiences and future directions. Expert Rev Cardiovasc Ther. 2017;15:481-9.

9. Provias T, Dudzinski DM, Jaff MR, Rosenfield K, Channick R, Baker J, et al. The Massachusetts general hospital pulmonary embolism response team (MGH PERT): creation of a multidisciplinary program to improve care of patients with massive and submassive pulmonary embolism. Hosp Pract (1995). 2014;42:31-7.

10. Rosovsky R, Borges J, Kabrhel C, Rosenfield K. Pulmonary embolism response team: inpatient structure, outpatient follow-up, and is it the current standard of care? Clin Chest Med. 2018;39:621-30.

11. Jerjes-Sánchez C, Cantu-Brito C, Arauz A, Martínez-Sánchez C, Sandoval-Zarate J, Ramirez-Rivera A, et al. Guías para anticoagulación y trombolisis del tromboembolismo venoso, infarto con elevación del st, cardioembolismo cerebral y del infarto cerebral agudo. Arch Cardiol Mex. 2017;87 Suppl 1:1-66

12. Dudzinski DM, Piazza G. Multidisciplinary pulmonary embolism response teams. Circulation. 2016:133:98-103.

13. Kabrhel C, Rosovsky R, Channick R, Jaff MR, Weinberg I, Sundt T, et al. A multidisciplinary pulmonary embolism response team: initial 30-month experience with a novel approach to delivery of care to patients with submassive and massive pulmonary embolism. Chest. 2016;150:384-93.

14. Jerjes-Sánchez C. Thrombolysis in pulmonary embolism. In: Jerjes-Sánchez C, editor. Thrombolysis in Pulmonary Embolism. $1^{\text {st }}$ ed. Cham, Switzerland: Springer International Publishing; 2015. p. 175-207.

15. Jerjes-Sánchez C, Villarreal-Umaña S, Ramírez-Rivera A, Garcia-Sosa A, Miguel-Canseco L, Archondo T, et al. Improving adjunctive treatment in pulmonary embolism and fibrinolytic therapy. The role of enoxaparin and weight-adjusted unfractionated heparin. J Thromb Thrombolysis. 2009;27:154-62

16. Jerjes-Sanchez C, Ramirez-Rivera A, Arriaga-Nava R, Iglesias-Gonzalez S, Gutierrez P, Ibarra-Perez C, et al. High dose and shortterm streptokinase infusion in patients with pulmonary embolism: prospective with seven-year follow-up trial. J Thromb Thrombolysis. 2001; 12:237-47.

17. Jerjes-Sánchez C, Ramírez-Rivera A, Arriaga-Nava R, Pimentel-Morales G. Dosis altas e infusión rápida de estreptoquinasa para el tratamiento de tromboembolia pulmonar masiva. Arch Cardiol Mex. 1993, 63:227-34.

18. Jerjes-Sánchez C, Gutiérrez-Fajardo $P$, Ramírez-Rivera $A$, García-Mollinedo ML, Hernández-Chávez G. Infarto agudo del miocardio del ventrículo derecho secundario a tromboembolia pulmonar masiva. Arch Cardiol Mex. 1995;65:65-73.

19. Jerjes-Sanchez C, Ramírez-Rivera A, de Lourdes García M, Arriaga-Nava R, Valencia S, Rosado-Buzzo A, et al. Streptokinase and heparin versus heparin alone in massive pulmonary embolism: a randomized controlled trial. J Thromb Thrombolysis. 1995;2:227-9.

20. Ramirez-Rivera A, Gutierrez-Fajardo $P$, Jerjes-Sánchez $C$, Hernández-Chávez G. Acute right myocardial infarction without significant obstructive coronary lesions secondary to massive pulmonary embolism. Chest. 1993;104:80

21. Silvain J, Collet JP, Nagaswami C, Beygui F, Edmondson KE, Bellemain-Appaix A, et al. Composition of coronary thrombus in acute myocardial infarction. J Am Coll Cardiol. 2011;57:1359-67.

22. O'Gara PT, Kushner FG, Ascheim DD, Casey DE Jr., Chung MK de Lemos JA, et al 2013 ACCF/AHA guideline for the management of ST-elevation myocardial infarction: a report of the American college of cardiology foundation/American heart association task force on practice guidelines. Circulation. 2013;127:e362-425

23. Powers WJ, Rabinstein AA, Ackerson T, Adeoye OM, Bambakidis NC Becker $\mathrm{K}$, et al 2018 guidelines for the early management of patients with acute ischemic stroke: a guideline for healthcare professionals from the American heart association/American stroke association. Stroke. 2018;49:e46-e110.

24. Ibanez B, James S, Agewall S, Antunes MJ, Bucciarelli-Ducci C Bueno $\mathrm{H}$, et al 2017 ESC guidelines for the management of acute myocardial infarction in patients presenting with ST-segment elevation: the task force for the management of acute myocardial infarction in patients presenting with ST-segment elevation of the European society of cardiology (ESC). Eur Heart J. 2018;39:119-77.

25. Rosenfield K, Rosovsky R. Multidisciplinary Care for Pulmonary Embolism. Mass General Advances in Motion; 2017. Available from: https:// www.advances. massgeneral.org/cardiovascular/article. aspx?id=1007.

26. Jaff MR, McMurtry MS, Archer SL, Cushman M, Goldenberg N, Goldhaber SZ, et al. Management of massive and submassive pulmonary embolism, iliofemoral deep vein thrombosis, and chronic thromboembolic pulmonary hypertension: a scientific statement from the American heart association. Circulation. 2011;123:1788-830.
27. Lee $\mathrm{JH}$, Kwun $\mathrm{WH}$, Suh BY. The results of aspiration thrombecomy in the endovascular treatment for iliofemoral deep vein thrombosis. J Korean Surg Soc. 2013;84:292-7.

28. Karahan O, Kutas HB, Gurbuz O, Tezcan O, Caliskan A, Yavuz C, et al. Pharmacomechanical thrombolysis with a rotator thrombolysis device in iliofemoral deep venous thrombosis. Vascular. 2016;24:481-6.

29. Garcia MJ, Lookstein R, Malhotra R, Amin A, Blitz LR, Leung DA, et al. Endovascular management of deep vein thrombosis with rheolytic thrombectomy: final report of the prospective multicenter PEARL (Peripheral use of angioJet rheolytic thrombectomy with a variety of catheter lengths) registry. J Vasc Interv Radiol. 2015;26:777-85.

30. Dumantepe M, Tarhan IA, Ozler A. Treatment of chronic deep vein thrombosis using ultrasound accelerated catheter-directed thrombolysis. Eur J Vasc Endovasc Surg. 2013;46:366-71.

31. Gasparis AP, Labropoulos N, Tassiopoulos AK, Phillips B, Pagan J, Cheng Lo, et al. Midterm follow-up after pharmacomechanical thrombolysis for lower extremity deep venous thrombosis. Vasc Endovascular Surg. 2009;43:61-8

32. Wang W, Sun R, Chen Y, Liu C. Meta-analysis and systematic review of percutaneous mechanical thrombectomy for lower extremity deep vein thrombosis. J Vasc Surg Venous Lymphat Disord. 2018;6:788-800.

33. Arko FR, Davis CM $3^{\text {rd }}$, Murphy EH, Smith ST, Timaran CH, Modrall JG, et al. Aggressive percutaneous mechanical thrombectomy of deep venous thrombosis: early clinical results. Arch Surg. 2007;142:513-8.

34. Jerjes-Sanchez C, Rodriguez D, Navarrete A, Parra-Cantu C Joya-Harrison J, Vazquez E, et al. Inferior vena cava filters in pulmonary embolism: a historic controversy. Arch Cardiol Mex. 2017;87:155-66.

35. Raju S, Davis M, Martin A. Assessment of residual thrombus after venous thrombolytic regimens. J Vasc Surg Venous Lymphat Disord. 2014;2:148- 54

36. Konstantinides SV, Torbicki A, Agnelli G, Danchin N, Fitzmaurice D, Galiè N, et al 2014 ESC guidelines on the diagnosis and management of acute pulmonary embolism. Eur Heart J. 2014;35:3033-69, 3069a-3069k.

37. Kearon C, Akl EA, Ornelas J, Blaivas A, Jimenez D, Bounameaux H, et al. Antithrombotic therapy for VTE disease: chest guideline and expert panel report. Chest. 2016;149:315-52

38. Guyatt GH, Akl EA, Crowther M, Gutterman DD, Schuünemann HJ, American College of Chest Physicians Antithrombotic Therapy and Prevention of Thrombosis Panel. et al. Executive summary: antithrombotic therapy and prevention of thrombosis, $9^{\text {th }}$ ed: American college of chest physicians evidence-based clinical practice guidelines. Chest. 2012;141:7S-47S

39. Meyer G, Vicaut E, Danays T, Agnelli G, Becattini C, Beyer-Westendorf J, et al. Fibrinolysis for patients with intermediate-risk pulmonary embolism. N Engl J Med. 2014;370:1402-11.

40. Sharifi M, Bay C, Skrocki L, Rahimi F, Mehdipour M, "MOPPET" Investigators. Moderate pulmonary embolism treated with thrombolysis (from the "MOPETT" Trial). Am J Cardiol. 2013;111:273-7.

41. Eid-Lidt G, Gaspar J, Sandoval J, de los Santos FD, Pulido T, González Pacheco $\mathrm{H}$, et al. Combined clot fragmentation and aspiration in patients with acute pulmonary embolism. Chest. 2008;134:54-60.

42. Tapson VF, Sterling K, Jones N, Elder M, Tripathy U, Brower J, et al. A randomized trial of the optimum duration of acoustic pulse thrombolysis procedure in acute intermediate-risk pulmonary embolism: the OP. TALYSE PE trial. JACC Cardiovasc Interv. 2018;11:1401-10.

43. Piazza G, Hohlfelder B, Jaff MR, Ouriel K, Engelhardt TC, Sterling KM, et al. A prospective, single-arm, multicenter trial of ultrasound-facilitated, catheter-directed, low-dose fibrinolysis for acute massive and submassive pulmonary embolism: the SEATTLE II study. JACC Cardiovasc Interv. 2015;8:1382-92

44. Kucher N, Boekstegers P, Müller OJ, Kupatt C, Beyer-Westendorf J, Heitzer T, et al. Randomized, controlled trial of ultrasound-assisted catheter-directed thrombolysis for acute intermediate-risk pulmonary embolism. Circulation. 2014:129:479-86.

45. Doyen D, Castellani M, Moceri P, Chiche O, Lazdunski R, Bertora D, et al. Patent foramen ovale and stroke in intermediate-risk pulmonary embolism. Chest. 2014;146:967-73.

46. Heit JA. The epidemiology of venous thromboembolism in the community. Arterioscler Thromb Vasc Biol. 2008;28:370-2.

47. Beckman MG, Hooper WC, Critchley SE, Ortel TL. Venous thromboembolism: a public health concern. Am J Prev Med. 2010:38:S495-501.

48. Mazzolai L, Aboyans V, Ageno W, Agnelli G, Alatri A, Bauersachs R, et al. Diagnosis and management of acute deep vein thrombosis: a joint consensus document from the European society of cardiology working groups of aorta and peripheral vascular diseases and pulmonary circulation and right ventricular function. Eur Heart J. 2018;39:4208-18.

49. Benin AL, Borgstrom CP, Jenq GY, Roumanis SA, Horwitz LI. Republished: defining impact of a rapid response team: qualitative study with nurses, physicians and hospital administrators. Postgrad Med J. 2012;88:575-82 